\title{
Aldosterone predicts major adverse cardiovascular events in patients with acute myocardial infarction
}

\author{
Matthew Fomonyuy Yuyun, ${ }^{1,2}$ Sandeep K Jutla, ${ }^{1,2}$ Paulene A Quinn, ${ }^{1,2}$ Leong L Ng ${ }^{1,2}$
}

'Department of Cardiovascular Sciences, University of Leicester, Leicester, UK 2Leicester NIHR Biomedical Research Unit in Cardiovascular Disease, Glenfield Hospital, Leicester, UK

\section{Correspondence to Professor Leong $\mathrm{L} \mathrm{Ng}$ Department of Cardiovascular Sciences, University of Leicester, Robert Kilpatrick Clinical Sciences Building, Leicester Royal Infirmary, Leicester LE2 7LX, UK; IIn1@leicester.ac.uk}

Accepted 22 July 2012

\begin{abstract}
Objective Aldosterone is associated with increased mortality in chronic heart failure patients and correlates with adverse outcomes after an acute myocardial infarction (AMI) in smaller cohorts. We evaluated the prognostic significance of plasma aldosterone in a large cohort of post-AMl patients in relation to major adverse cardiovascular events (MACE).

Design A prospective cohort study.

Setting University Hospitals of Leicester, UK.

Patients Consecutive 955 patients admitted with AMI. Plasma aldosterone levels were measured in these patients.
\end{abstract}

Main outcome measures During the 2 years followup, MACE which was a composite of all-cause mortality, myocardial reinfarction, and hospitalisation for heart failure as well as secondary endpoints (all-cause mortality and a combination of all-cause mortality and hospitalisation for heart failure), were ascertained.

Results MACE occured in $\mathrm{N}=261,27.3 \%$, all-cause mortality $(N=114,11.9 \%)$ and a combination of all-cause mortality and hospitalisation for heart failure $(\mathrm{N}=176,18.4 \%)$. Patients with MACE had significantly higher median levels of aldosterone than those without (1150.1 vs $950.4 \mathrm{pmol} / \mathrm{l}, \mathrm{p}=0.0118$ ). The multivariate adjusted $\mathrm{HR}(95 \% \mathrm{Cl})$ for log aldosterone on MACE was 1.26 (1.01 to 1.56 ), $p=0.041$; all-cause mortality 1.60 (1.13 to 2.27), $p=0.008$; and combination of all-cause mortality and heart failure 1.50 (1.14 to 1.97), $p=0.003$. Conclusions The prognostic significance of aldosterone for a variety of endpoints in this large cohort of post-AMI patients is not new and adds to the findings by others. The magnitude of the increase in aldosterone secretion post infarction is higher than previously believed.

\section{INTRODUCTION}

Mortality from coronary artery disease (CAD) has declined over the past decades in developed countries. This is largely due to a decrease in incidence accounted for by primary prevention and a decrease in case-fatality rate accounted for by high-impact interventional, surgical and pharmacological therapies, other secondary prevention measures and improved coronary care facilities. ${ }^{1}{ }^{2}$ Nevertheless the burden of disease remains high and $\mathrm{CAD}$ is still the leading cause of death in developed countries accounting for about $15-20 \%$ of all deaths, and its incidence is rising in developing countries. ${ }^{1-3}$

Risk stratification post acute myocardial infarction (AMI) with multiple risk predictors including biomarkers with pathophysiologically distinct pathways appear to be the way forward. Hormones acting on the mineralocorticoid receptor are associated with adverse cardiovascular disease (CVD) outcomes. ${ }^{4-7}$ Much evidence has accrued in the past two decades to support the deleterious effects of aldosterone and cortisol activation of this receptor, and the role of prereceptor enzymes which determine the relative concentrations of these two hormones available at different tissues for mineralocortocid receptor binding has emerged. ${ }^{4-12}$

Aldosterone is associated with hypertension, ${ }^{13}$ endothelial dysfunction, ${ }^{14}$ atherosclerosis, ${ }^{4}{ }^{5}$ poor systemic vascular compliance, increased vascular tone and decreased baroreceptor sensitivity, and promotion of myocardial necrosis and increase in infarct size. ${ }^{5}{ }^{15}$ Aldosterone is also associated with increased risk of death and acute ischaemic events in $\mathrm{CAD}$ patients with a preserved left ventricular function and no AMI, ${ }^{16}$ increased mortality post myocardial infarction. ${ }^{10-12}$ increased cardiac remodelling post myocardial necrosis ${ }^{17} 18$ and increased mortality in chronic heart failure patients. ${ }^{8} 9$ Plasma aldosterone/renin concentration ratio is strongly associated with blood pressure values in patients undergoing coronary angiography. ${ }^{19}$ Trials have shown that mineralocorticoid blockade in chronic heart failure (RALES), ${ }^{20}$ and post AMI with ventricular dysfunction (EPHESUS) reduced mortality and hospitalisation for heart failure. ${ }^{21}$

Recent studies have demonstrated that aldosterone correlates with poor outcomes in patients undergoing scheduled coronary angiography, ${ }^{22}$ and in post-AMI patients. ${ }^{10-12}$ Whether aldosterone is predictive of major adverse cardiovascular events (MACE) in a larger cohort of post-AMI patients remains to be clearly shown. Therefore our aim was to prospectively evaluate the prognostic significance of plasma aldosterone in a large cohort of post-AMI patients in relation to MACE. We also assessed whether it correlated with secondary endpoints which were all-cause mortality, and combination all-cause mortality and hospitalisation for heart failure.

\section{MATERIALS AND METHODS \\ Study design and study population}

In this prospective cohort study, 955 consecutive patients with AMI admitted to the University Hospitals of Leicester NHS Trust between 2003 and 2008 were recruited. Written informed consent was obtained from patients, and the study complied with the Declaration of Helsinki and was approved by the local ethics committee. AMI was diagnosed if a patient had a plasma cardiac troponin I level $>0.1 \mathrm{ng} / \mathrm{ml}$ with at least one of the following: chest pain lasting $>20 \mathrm{~min}$ or diagnostic serial ECG changes consisting of new pathological $\mathrm{Q}$ waves or ST segment and $\mathrm{T}$ wave changes. Exclusion criteria included malignancy or 
surgery in the previous month. Data on demographics, clinical treatment, laboratory and echocardiography were obtained. All patients received standard secondary prevention therapy for ischaemic heart disease, and revascularization was at the discretion of the attending physician.

\section{Blood sampling}

Blood samples were drawn at 3-5 days after admission with AMI. $10 \mathrm{ml}$ of venous blood was taken from the antecubital vein into tubes containing EDTA $(1 \mathrm{ml} / 10 \mathrm{ml}$ blood) and aprotinin (500 international units $/ \mathrm{ml}$ blood). The blood samples were centrifuged at $3000 \mathrm{rpm} 4^{\circ} \mathrm{C}$ for $15 \mathrm{~min}$. The plasma was aspirated and stored at $-80^{\circ} \mathrm{C}$ until assayed.

\section{Steroid extraction}

Plasma samples were defrosted at room temperature. $100 \mu \mathrm{l}$ of patients' plasma was mixed with $1 \mathrm{ml}$ dichloromethane. The mixture was left on a shaker for $2 \mathrm{~h}$ and then spun down using a centrifuge at $2000 \mathrm{rpm}$. Then $0.7 \mathrm{ml}$ of the dichloromethane layer was aspirated, and dried down in vacuum centrifuge to leave the aldosterone extract.

\section{Aldosterone immunoassay}

Aldosterone was measured using a competitive immunoluminometric assay. Each well of the microlite-two plate was coated with $100 \mu \mathrm{l}$ (10 ng per well) of sheep antibody (Abcam) and left overnight at room temperature. Then $50 \mu$ l of polyclonal aldosterone antibody (Abcam) was added into each well and the samples were left to incubate overnight at $4^{\circ} \mathrm{C}$. Using the automatic washer, the plates were washed in wash buffer and $200 \mu \mathrm{l}$ of bovine serum albumin (BSA) was pipetted into each well of the plate. The plates were left for $2 \mathrm{~h}$ in BSA at room temperature, and then washed in wash buffer to remove any unbound BSA. Following this, $100 \mu \mathrm{l}$ of sample was pipetted accurately into the well, each sample done in duplicate format and $50 \mu \mathrm{l}$ of aldosterone-biotin tracer was pipetted into each well. The plates were left for incubation overnight at $4^{\circ} \mathrm{C}$ and the plates were washed in wash buffer to remove any unbound tracer. Then $100 \mu \mathrm{l}$ of MAE-streptavidin was pipetted into each well and the plates were left in the dark at room temperature to incubate for $90 \mathrm{~min}$. The plates were washed in wash buffer to remove unbound MAE-streptavidin. The plates were read using chemiluminescence in a DYNEX MLX luminometer (Dynex Technologies, Worthing, West Sussex, UK) and plate readings were given in relative light units (RLU). A standard curve was constructed using Rodbard's quadratic equation regression analysis (Biosoft, Great Shelford, Cambridge, UK). The luminometer readings were plotted on the $y$-axis (RLU) against aldosterone concentration ( $\mathrm{pmol} / \mathrm{l}$ ) on the $\mathrm{x}$-axis. The patient's plasma aldosterone concentration was calculated by interpolation of the standard curve. The RLU values for the samples were plotted on the standard curve and the corresponding concentration in pmol/1 was efficiently calculated. The reproducibility of the assay was assessed by calculating the coefficient of variation which was $4.1 \%$.

\section{NT-proBNP assay}

Plasma NT-proBNP assay was measured using a non-competitive immunoluminometric assay as previously published. ${ }^{23}$ The lower limit of detection was $0.3 \mathrm{pmol} / \mathrm{l}$.

\section{Endpoints}

Patients were reviewed 24 months after their initial admission with AMI, giving a censoring time of 730 days. The primary endpoint was MACE, which was a composite of all-cause mortality, myocardial reinfarction, and hospitalisation for heart failure. There were two secondary endpoints: all-cause mortality and a combination of all-cause mortality and hospitalisation for heart failure. Deaths were validated by reviewing the hospital record management systems and the Office of National Statistics Registry. Hospitalisation for heart failure was defined as a hospital admission for which heart failure was the primary reason, requiring treatment with high-dose diuretics, inotropes or intravenous nitrate with echocardiographic evidence of heart failure. New myocardial infarction was defined as a plasma cardiac troponin I level $>0.1 \mathrm{ng} / \mathrm{ml}$ with at least one of the following: chest pain lasting $>20$ min or diagnostic serial ECG changes consisting of new pathological $\mathrm{Q}$ waves or ST segment and $T$ wave changes. New myocardial infarction and heart failure endpoints were ascertained using computerised hospital patients records for University Hospitals of Leicester and validated by contacting each patient.

\section{Statistical anlyses}

Statistical analyses were done using STATA V.11. The twosample $t$ test was used to test for any statistically signifcant difference between the means of normally distributed continuous variables across the binary outcomes (presence or absence of MACE, all-cause mortality, and combination of all-cause mortality and heart failure). The median and IOR were used for serum aldosterone because its distribution was positively skewed and for other skewed variables. The two-sample Wilcoxon rank-sum test was used to test for any significant difference in the medians of continuous variables which were not normally distributed across the binary outcomes. The Pearson $\chi^{2}$ test was use to compare categorical variables. The $\chi^{2}$ test for trend was used to test the trend of event rate across ordered quartiles of serum aldosterone. Curves of Kaplan-Meier survival estimates were obtained and the Logrank test was used to compare survival rates between patients whose aldosterone levels were above the median value and those below the median. Univariate and multivariate HRs for the predictive impact of baseline serum aldoesterone on endpoints were estimated by the Cox proportional hazard model. Because aldosterone distribution was skewed to the right, its levels were log-transformed to base 10 before the Cox regression analyses were carried out. The Likelihood ratio $\chi^{2}$ test was used to test for statistical significance of each variable in the Cox regression model.

\section{RESULTS}

\section{Baseline characteristics}

Table 1 shows baseline characteristics of the study participants in the total study population and stratified by MACE outcomes. Post-AMI patients who developed MACE were significantly older, and had significantly greater median levels of baseline serum aldosterone. Similar baseline distributions were seen when the baseline characteristics were stratified by presence or absence of secondary outcomes which which were allcause mortality or combination of all-cause mortality and heart failure binary outcomes.

\section{Major cardiovascular events rate}

The mean outcome was MACE which was a composite of myocardial infarction, death and hospitalisation for heart failure. During the 2 years of follow-up of the 955 post-AMI patients, MACE were $n=261,27.3 \%$. The secondary outcomes included all-cause mortality $(n=114,11.9 \%)$ and a combination 
Table 1 Baseline characteristics of the entire study population and according to the presence or absence of MACE, which was the composite endpoint

\begin{tabular}{|c|c|c|c|c|}
\hline Variables & Total & MACE absent & MACE present & p Value \\
\hline Number & 955 & $694(72.7)$ & $261(27.3)$ & \\
\hline Aldosterone $(\mathrm{pmol} / \mathrm{l})^{*}$ & 997.3 (499.3-1785.7) & $950.4(474.3-1700.0)$ & 1150.1 (578.1-2064.2) & 0.0118 \\
\hline Age & $66.5(12.8)$ & $64.3(12.4)$ & $72.3(11.7)$ & $<0.0001$ \\
\hline Male & $667(69.8 \%)$ & $510(73.5 \%)$ & $157(60.2 \%)$ & $<0.001$ \\
\hline Female & $288(30.2 \%)$ & $184(26.5 \%)$ & $104(39.9 \%)$ & $<0.001$ \\
\hline Hypertension & $512(53.6 \%)$ & $344(49.6 \%)$ & $168(64.4 \%)$ & $<0.001$ \\
\hline Diabetes & $244(25.6 \%)$ & $159(22.9 \%)$ & $85(32.6 \%)$ & 0.002 \\
\hline Previous CAD & $356(37.3 \%)$ & $230(33.1 \%)$ & $126(48.3 \%)$ & $<0.001$ \\
\hline Smoking history & $410(43.0 \%)$ & 318 (45.9\%) & $92(35.4 \%)$ & 0.004 \\
\hline STEMI & $398(41.7 \%)$ & $285(41.1 \%)$ & $113(43.3 \%)$ & 0.534 \\
\hline eGFR $\left(\mathrm{ml} / \mathrm{min} / 1.73 \mathrm{~m}^{2}\right)$ & $66.2(19.9)$ & $69.4(18.8)$ & $57.6(20.6)$ & $<0.0001$ \\
\hline Killip Class >1 & $376(39.6 \%)$ & $225(32.7 \%)$ & $151(58.1 \%)$ & $<0.001$ \\
\hline Heart failure & $41(4.3 \%)$ & $28(4.0 \%)$ & $13(5.0 \%)$ & 0.520 \\
\hline NTproBNP(pmol/l)* & $768.4(255.2-2259.9)$ & 591.2 (182.2-1591.1) & $2036.1(568.8-3908.4)$ & $<0.0001$ \\
\hline ACEI or ARB & $766(80.2 \%)$ & $576(83.0 \%)$ & $190(72.8 \%)$ & $<0.001$ \\
\hline Betablockers & $757(79.3 \%)$ & $574(82.7 \%)$ & $183(70.1 \%)$ & $<0.001$ \\
\hline Diuretics & $244(25.6 \%)$ & $141(20.3 \%)$ & $103(39.7 \%)$ & $<0.001$ \\
\hline Statins & 811 (84.9\%) & 614 (88.5\%) & 197 (75.5\%) & $<0.001$ \\
\hline Aspirin & $803(84.0 \%)$ & 601 (86.6\%) & 202 (77.4\%) & 0.001 \\
\hline GRACE score & $151.2(39.8)$ & $143.6(36.6)$ & $171.9(41.1)$ & $<0.0001$ \\
\hline
\end{tabular}

Values are mean (SD) or number (\%), except for aldosterone and NTproBNP which are median and IQR.

${ }^{*}$ Median and IQR.

ARB, angiotensin receptor blocker; CAD, coronary artery disease; eGFR, estimated glomerular filtration rate; MACE, major adverse cardiovascular events; NTproBNP, N-terminal pro-B type natriuretic peptide; STEMI, ST elevation myocardial infarction; GRACE, global registry of acute coronary events.

of all-cause mortality and hospitalisation for heart failure $(n=176,18.4 \%)$.

\section{Aldosterone and MACE}

Patients with MACE had significantly higher median levels of aldosterone than those without (1150.1 vs $950.4 \mathrm{pmol} / \mathrm{l}$, $\mathrm{p}=0.0118$ ). As shown in table $2, \log 10$-transformed aldosterone levels were independently predictive of future MACE after adjusting for covariates listed below the table. As shown in figure $1 \mathrm{~A}$, the MACE rate increased across ordered quartiles of baseline aldosterone $\left(\chi^{2}\right.$ test for trend of survivor functions across aldosterone quartiles $=9.17, p=0.0025$ ). Patients in the highest quartile of aldosterone had signficantly more risk of MACE compared to those in the lowest quartile with a HR (95\% CI) of 1.46 (1.01 to 2.12), p value 0.043 (table 2). Figure 2A shows statistically significant lower Kaplan-Meier survival estimates for MACE in patients with serum aldosterone above the median value compared to those below the median (Logrank test $\chi^{2}$ for adjusted survival $=4.60, p=0.0320$ ).

\section{Aldosterone and all-cause mortality}

All-cause mortality was experienced at a higher rate $(n=73$, $15.3 \%$ ) in patients with aldosterone levels above the median, compared to patients with aldosterone levels below the median $(\mathrm{n}=41,8.6 \%), \mathrm{p}=0.001$. Looking at this from a different angle, patients who died had signficantly higher median levels (IOR) of serum aldosterone of $1298.1 \mathrm{pmol} / 1$ (722.8 to 2338.8), compared to those who survived $959.7 \mathrm{pmol} / 1$ (481.7 to 1706.9), $\mathrm{p}=0.0005$. Figure $1 \mathrm{~B}$ shows death rates across quartiles of aldosterone. Table 2 depicts that log10-transformed aldosterone levels were independently predictive of death in a multivariate Cox model, alongside age, $\mathrm{N}$-terminal pro-B type natriuretic peptide, ACE inhibitors or angiotensin receptor blockers, $\beta$ blockers, and statins, in the 955 patients with AMI. Compared with the lowest quartile of aldosterone, patients in the highest quartile were at a significant risk of mortality with a HR
(95\% CI) of 2.21 (1.21 to 4.03), $\mathrm{p}=0.010$ (table 2). KaplanMeier survival estimates above and below median values of aldosterone are shown in figure $2 \mathrm{~B}$.

\section{Aldosterone and combination of death and hospitalisation for heart failure}

Median levels (IOR) of serum aldosterone were significantly higher in patients who experienced this combination (table 1). Looking at this from a different perspective, a higher rate ( $n=110,23.0 \%)$ of this combined outcome was experienced by patients with serum aldosterone levels above the median compared to those below the median ( $n=66,13.8 \%), p<0.001$. As shown in table 2, log10-transformed aldosterone levels were an independent predictor of the combination of death and heart failure. There was an increasing trend of events rates across ordered quartile of serum aldosterone with $\chi^{2}$ for trend of 19.56, $p<0.0001$. Compared to the lowest quartile, patients in the highest quartile had HRs (95\% CI) of 2.06 (1.29 to 3.28), $\mathrm{p}=0.002$, after controlling for confounding variables. Figures $1 \mathrm{C}$ and 2C depict mortality and Kaplan-Meier survival estimates respectively across quartiles of serum aldosterone.

\section{DISCUSSION}

Our study demonstrated that plasma aldosterone measured 3-5 days after occurence of myocardial infarction (ST elevation myocardial infarction (STEMI) and non-ST elevation myocardial infarction (NSTEMI) is independently predictive of MACE defined as all-cause mortality, reinfarction and hospitalisation with heart failure as well as secondary outcomes which were all-cause mortality alone and combination of all-cause mortality and hospitalisation for heart failure. These findings are similar to those observed in three previous publications, ${ }^{10-12}$ albeit in a larger cohort of patients and with different combinations of outcomes. Palmer et al had previously observed that plasma aldosterone levels post-AMI were independent predictors of survival and hospitalisation for heart failure over a 
Table 2 HRs (95\% Cl) from Cox regression for MACE, death, and death and heart failure, associated with Log aldosterone and upper quartiles compared to the lowest quartile of these variables, in post-acute myocardial infarction patients $(\mathrm{N}=955)$

\begin{tabular}{|c|c|c|c|}
\hline & Univariate & Multivariate & $\begin{array}{l}\text { p Values } \\
\text { (adjusted) }\end{array}$ \\
\hline \multicolumn{4}{|l|}{ MACE } \\
\hline Log aldosterone & $1.39(1.13$ to 1.72$)$ & $1.26(1.01$ to 1.56$)$ & 0.041 \\
\hline First quartile & Reference & Reference & \\
\hline Second quartile & $1.42(0.99$ to 2.05$)$ & $1.28(0.87$ to 1.87$)$ & 0.210 \\
\hline Third quartile & $1.39(0.96$ to 2.01$)$ & $1.23(0.81$ to 1.83$)$ & 0.343 \\
\hline Fourth quartile & 1.75 (1.23 to 2.51$)$ & 1.46 (1.01 to 2.12$)$ & 0.043 \\
\hline \multicolumn{4}{|c|}{ All-cause mortality } \\
\hline Log aldosterone & $1.82(1.32$ to 2.50$)$ & $1.60(1.13$ to 2.27$)$ & 0.008 \\
\hline First quartile & Reference & Reference & \\
\hline Second quartile & $1.60(0.86$ to 2.99$)$ & $1.30(0.68$ to 2.50$)$ & 0.425 \\
\hline Third quartile & 2.17 (1.20 to 3.95$)$ & $1.45(0.75$ to 2.79$)$ & 0.273 \\
\hline Fourth quartile & $2.72(1.5$ to 4.86$)$ & 2.21 (1.21 to 4.03$)$ & 0.010 \\
\hline \multicolumn{4}{|c|}{ All-cause mortalilty and hospitalisation for heart failure } \\
\hline Log aldosterone & $1.66(1.29$ to 2.15$)$ & $1.50(1.14$ to 1.97$)$ & 0.003 \\
\hline First quartile & Reference & Reference & \\
\hline Second quartile & $1.40(0.86$ to 2.27$)$ & $1.34(0.80$ to 2.25$)$ & 0.272 \\
\hline Third quartile & 1.87 (1.17 to 2.97 ) & 1.64 (0.97 to 2.78$)$ & 0.064 \\
\hline Fourth quartile & 2.46 (1.56 to 3.85$)$ & 2.06 (1.29 to 3.28$)$ & 0.002 \\
\hline
\end{tabular}

Multivariate HRs were adjusted for age, sex, history of diabetes, hypertension, smoking, Killip class or heart failure, eGFR, NTproBNPd, and ACE inhibitors or angiotensin receptor blockers, $\beta$ blockers, statins, and diuretics.

eGFR, estimated glomerular filtration rate; MACE, major adverse cardiovascular events.

5 -year follow-up in their cohort of $>500$ patients. Beygui et al had earlier observed that in STEMI patients referred for primary percutaneous angioplasty, high aldosterone levels correlated with an increase in rates of inhospital all-cause death, cardiovascular death, heart failure, ventricular fibrillation, resuscitated cardiac arrest and increased mortality after 6 months of follow-up $>300$ patients. ${ }^{11}$ They went further to demonstrate in the OPERA study that high plasma levels of aldosterone were independently predictive of the composite outcome of death, resuscitated cardiac arrest, recurrent myocardial infarction, heart failure and stroke in $>400$ post-AMI patients during hospitalisation and after 1 year of follow-up. ${ }^{12}$ Recently, Ivanes et al observed a significantly increased risk of death and acute ischaemic events in CAD patients with a preserved left ventricular function and no AMI with high levels of baseline aldosterone. ${ }^{16}$ More recently Weir et al showed that in post-AMI patients, levels of cortisol and aldosterone were independently predictive of cardiac remodelling defined as change in left ventricle (LV) end-systolic volume index, measured using contrast-enhanced cardiac magnetic resonance at baseline and after 24 weeks of follow-up. ${ }^{17}$ In patients undergoing scheduled coronary angiography in the large Ludwigshafen risk and cardiovascular health (LURIC) Study, variation in levels of plasma aldosterone concentration within the normal range was associated with increased risk of all-cause and CVD mortality independent of major CVD risk factors, and this risk persisted in a subgroup of patients who had suffered an acute coronary syndrome. $^{22}$ The importance of aldosterone in the mediation of adverse cardiovascular outcomes has been underlined by two landmark trials, randomised aldactone evaluation study (RALES) and eplerenone post -acute myocardial infarction heart failure efficacy and survival study (EPHESUS). ${ }^{20} 21$ In the RALES trial, mineralocorticoid receptor blockade with spironolactone in addition to standard therapy substantially reduced the risk of both death and hospitalisation among patients with
1A. MACE rate $(95 \% \mathrm{Cl})$ across aldosterone quartiles
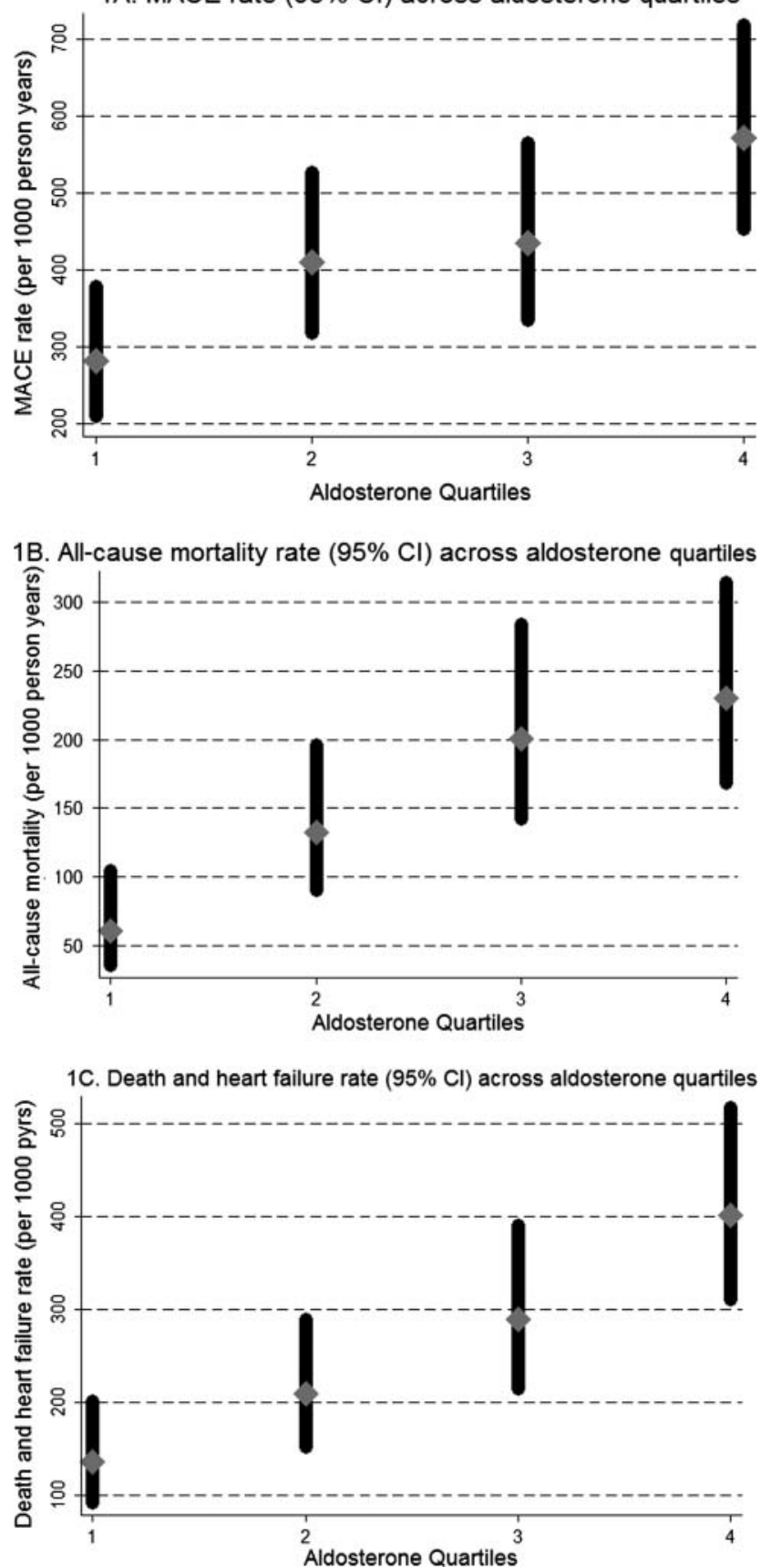

Figure 1 Outcomes across quartiles of plasma aldosterone major adverse cardiovascular events rate per 1000 person-years (A), all-cause mortality $(B)$, and combination of all-cause mortality and hospitalisation for heart failure $(C)$, across quartiles of aldosterone in post-acute myocardial infarction patients $(\mathrm{N}=955) \cdot \chi^{2}$ test for trend of survivor functions across aldosterone quartiles for these three outcomes are respectively $9.17(p=0.0025), 14.04(p=0.0002)$, and $19.56(p<0.001)$.

severe heart failure. ${ }^{20}$ In the EPHESUS trial, eplerenone added to optimal medical therapy reduced morbidity and mortality among patients with AMI complicated by left ventricular dysfunction and heart failure, ${ }^{21}$ and an earlier initiation of eplerenone was associated with a greater reduction in adverse outcomes. $^{24}$

In both the studies by Palmer et al and Beygui et al, the mean level of aldosterone was about 100-120 pmol/1 (which are within the normal range), whereas in our study the levels 

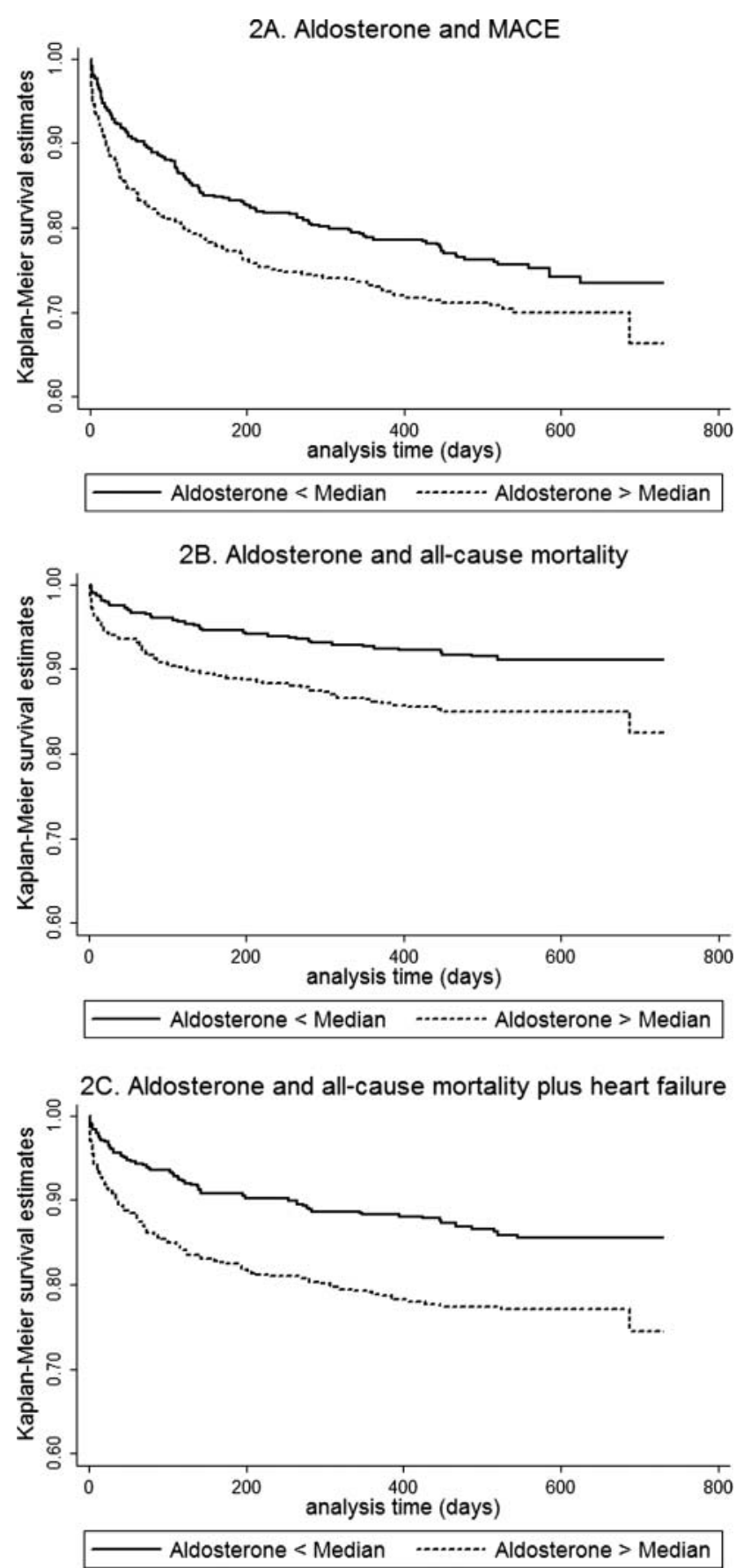

Figure 2 Plasma aldosterone and Kaplan-Meier survival curves for major adverse cardiovascular events (MACE) (A), all-cause mortality (B), and combination of all-cause mortality and hospitalisation for heart failure $(\mathrm{C})$ by ordered categories (below and above the median) of baseline plasma aldosterone in post-acute myocardial infarction patients $(\mathrm{N}=955)$. Logrank test $\chi^{2}=4.60, p=0.0320$ (MACE); $10.93, p=0.0009$ (all-cause mortality); 14.98, $\mathrm{p}=0.0001$ (combination of death and hospitalisation for heart failure).

of aldosterone measured 3-5 days post infarction were significantly much higher, around the $1000 \mathrm{pmol} / \mathrm{l}$ mark and higher than the quoted normal range of $100-450 \mathrm{pmol} / \mathrm{l}$ in recumbent resting adults. Our data suggests that activation of aldosterone secretion post infarction may be more marked than previously believed, and underscores the importance of effective mineralocorticoid blockade in these patients.
Aldosterone is normally produced in the adrenal zona glomerulosa via activation of the renin angiotensin aldosterone system (RAAS) pathway. However, extra-adrenal aldosterone expression has been observed in cardiomyocytes, ${ }^{5}{ }^{25}$ brain $^{5} 26$ and vascular smooth muscle. ${ }^{5}$ Aldosterone acts on mineralocorticoid receptors which are expressed both on sodiumtransporting epithelia and vascular endothelium, and non-epithelial tissues such as brain, ${ }^{28}$ myocardium ${ }^{52}$ and vascular smooth muscle cells. ${ }^{50}$ It has been reported that plasma aldosterone is extracted through the heart in patients with chronic heart failure and in post-AMI patients and that the transcardiac gradient of plasma aldosterone correlates with LV end-diastolic volume index and plasma levels of procollagen type III aminoterminal peptide, a marker of myocardial fibrosis and that administration of mineralocorticoid receptor blockade prevents this remodelling. ${ }^{18} 31$ Other studies have shown that aldosterone is predictive of mortality in chronic heart failure patients, alongside other neuroendocrine markers. ${ }^{9}$ It is well established that in the Conn's or primary aldosteronism, hypertension is induced. ${ }^{13}$

The possible mechanisms through which aldosterone adversely affects the cardiovascular system are legion. These include increased sodium retention associated with consequential fluid overload, hypertension and potentially arrhythmogenic potassium and magnesium depletion, ${ }^{6} 32$ the inhibition of nitric oxide, ${ }^{33}$ oxidative stress, ${ }^{34}$ endothelial dysfunction ${ }^{14}$ and atherosclerosis. Aldosterone has also been associated with poor systemic vascular compliance, increased vascular tone and decreased baroreceptor sensitivity, ${ }^{35}$ promotion of myocardial necrosis and increase in infarct size,${ }^{15}$ cardiac fibroblast activation and collagen deposition, ${ }^{36}$ cardiac myocyte hypertrophy, myocardial fibrosis and overall remodelling, ${ }^{6} 18$ and arrhythmias and sudden cardiac death. ${ }^{12} 21$ Plasma aldosterone/renin concentration ratios are strongly predictive of blood pressure values in patients undergoing coronary angiography. ${ }^{19}$

\section{LIMITATIONS}

Plasma concentrations of aldosterone before and during the ischaemic event were unknown. The plasma levels were measured 3-5 days after the index infarction and we were therefore unable to observe any differences in aldosterone levels over the course of the event. However our data was representative of the acute phase of AMI, where early interventional therapies based on severity of disease would be most appropriate. During follow-up, we did not ascertain the number of patients in this cohort who needed target vessel revascularisation as an endpoint, which could have increased the number of MACE.

\section{CONCLUSION}

Overall, aldosterone has been shown to be predictive of MACE. These results are not new but add to recent findings by others, albeit in a larger cohort of patients. Consideration of trials of early initiation of aldosterone blocking agents in patients who have suffered an AMI irrespective of whether they developed ventricular dysfunction is warranted.

Acknowledgements We thank all the patients who participated in the study, and members of the Pharmacology and Therapeutics Group for their contribution in patient recruitment and assaying.

Contributors Each author contributed fully to the work.

Funding This study was supported by the Leicester NIHR Biomedical Research Unit in Cardiovascular Disease and by the Van Geest Foundation.

Competing interest None. 
Patient consent Obtained.

Ethics approval Local Ethics Committee.

Provenance and peer review Not commissioned; externally peer reviewed

\section{REFERENCES}

1. Tunstall-Pedoe H, Kuulasmaa K, Mahonen M, et al. Contribution of trends in survival and coronary-event rates to changes in coronary heart disease mortality: 10-year results from 37 WHO MONICA project populations. Monitoring trends and determinants in cardiovascular disease. Lancet 1999;353:1547-57.

2. Roger VL, Go AS, Lloyd-Jones DM, et al. Heart disease and stroke statistics2011 update: a report from the American Heart Association. Circulation 2011;123: e18-209.

3. Murray CJ, Lopez AD. Global mortality, disability, and the contribution of risk factors: global burden of disease study. Lancet 1997;349:1436-42.

4. Funder JW. Mineralocorticoid receptors: distribution and activation. Heart Fail Rev 2005;10:15-22.

5. Chai $\mathbf{W}$, Danser $\mathrm{AH}$. Why are mineralocorticoid receptor antagonists cardioprotective? Naunyn-Schmiedeberg Arch Pharmacol 2006;374:153-62.

6. Connell JM, Davies E. The new biology of aldosterone. J Endocrinol 2005;186:1-20.

7. Walker BR. Glucocorticoids and cardiovascular disease. Eur J Endocrino/2007;157:545-59.

8. Yamaji M, Tsutamoto T, Kawahara $\mathrm{C}$ et al Serum cortisol as a useful predictor of cardiac events in patients with chronic heart failure: the impact of oxidative stress. Circ Heart Fail 2009;2:608-15.

9. Guder G, Bauersachs J, Frantz S, et al. Complementary and incremental mortality risk prediction by cortisol and aldosterone in chronic heart failure. Circulation 2007;115:1754-61.

10. Palmer BR, Pilbrow AP, Frampton CM, et al. Plasma aldosterone levels during hospitalization are predictive of survival post-myocardial infarction. Eur Heart $J$ 2008;29:2489-96.

11. Beygui F, Collet JP, Benoliel JJ, et al. High plasma aldosterone levels on admission are associated with death in patients presenting with acute ST-elevation myocardial infarction. Circulation 2006;114:2604-10.

12. Beygui F, Montalescot G, Vicaut E, et al. Aldosterone and long-term outcome after myocardial infarction: a substudy of the French nationwide observatoire sur la prise en charge hospitaliere, l'evolution a un an et les caracteristiques de patients presentant un infarctus du myocarde avec ou sans onde 0 (OPERA) study. Am Heart J 2009:157:680-7.

13. Calhoun DA. Aldosteronism and hypertension. Clin J Am Soc Nephrol 2006;1:1039-45.

14. Farquharson CA, Struthers AD. Aldosterone induces acute endothelial dysfunction in vivo in humans: evidence for an aldosterone-induced vasculopathy. Clin Sci 2002;103:425-31.

15. Oestreicher EM, Martinez-Vasquez D, Stone JR, et al. Aldosterone and not plasminogen activator inhibitor-1 is a critical mediator of early angiotensin II/ NG-nitro-L-arginine methyl ester-induced myocardial injury. Circulation 2003;108:2517-23.

16. Ivanes F, Susen S, Mouquet F, et al. Aldosterone, mortality, and acute ischaemic events in coronary artery disease patients outside the setting of acute myocardia infarction or heart failure. Eur Heart J 2011;33:191-202.
17. Weir RA, Tsorlalis IK, Steedman T, et al. Aldosterone and cortisol predict medium-term left ventricular remodelling following myocardial infarction. Eur $\mathrm{J}$ Heart Fail 2011;13:1305-13

18. Hayashi M, Tsutamoto T, Wada A, et al. Relationship between transcardiac extraction of aldosterone and left ventricular remodeling in patients with first acute myocardial infarction: extracting aldosterone through the heart promotes ventricular remodeling after acute myocardial infarction. J Am Coll Cardiol 2001;38:1375-82.

19. Tomaschitz A, Maerz W, Pilz S, et al. Aldosterone/renin ratio determines peripheral and central blood pressure values over a broad range. J Am Coll Cardiol 2010;55:2171-80

20. Pitt B, Zannad F, Remme WJ, et al. The effect of spironolactone on morbidity and mortality in patients with severe heart failure. Randomized aldactone evaluation study investigators. N Engl J Med 1999;341:709-17.

21. Pitt B, Remme W, Zannad F, et al. Eplerenone, a selective aldosterone blocker, in patients with left ventricular dysfunction after myocardial infarction. N Engl J Med 2003;348:1309-21.

22. Tomaschitz A, Pilz S, Ritz E, et al. Plasma aldosterone levels are associated with increased cardiovascular mortality: the Ludwigshafen Risk and Cardiovascular Health (LURIC) study. Eur Heart J 2010;31:1237-47.

23. Omland T, Persson A, Ng L, et al. N-terminal pro-B-type natriuretic peptide and long-term mortality in acute coronary syndromes. Circulation 2002;106:2913-18.

24. Adamopoulos C, Ahmed A, Fay R, et al. Timing of eplerenone initiation and outcomes in patients with heart failure after acute myocardial infarction complicated by left ventricular systolic dysfunction: insights from the EPHESUS trial. Eur J Heart Fail 2009:11:1099-105.

25. Silvestre JS, Robert V, Heymes C, et al. Myocardial production of aldosterone and corticosterone in the rat. Physiological regulation. J Biol Chem 1998:273:4883-91.

26. Gomez-Sanchez EP. Brain mineralocorticoid receptors: orchestrators of hypertension and end-organ disease. Curr Opin Nephrol Hypertens 2004;13:191-6.

27. Takeda Y, Miyamori I, Yoneda T, et al. Production of aldosterone in isolated rat blood vessels. Hypertension 1995;25:170-3.

28. de Kloet ER, Van Acker SA, Sibug RM, et al. Brain mineralocorticoid receptors and centrally regulated functions. Kidney Int 2000:57:1329-36.

29. Lombes M, Alfaidy N, Eugene E, et al. Prerequisite for cardiac aldosterone action. Mineralocorticoid receptor and 11 beta-hydroxysteroid dehydrogenase in the human heart. Circulation 1995;92:175-82.

30. Oberleithner H. Aldosterone makes human endothelium stiff and vulnerable. Kidney Int 2005;67:1680-2.

31. Nagata K, Obata K, Xu J, et al. Mineralocorticoid receptor antagonism attenuates cardiac hypertrophy and failure in low-aldosterone hypertensive rats. Hypertension 2006:47:656-64.

32. Weber KT, Sun Y, Wodi LA, et al. Toward a broader understanding of aldosterone in congestive heart failure. J Renin Angiotensin Aldosterone Syst 2003;4:155-63.

33. Ikeda U, Kanbe T, Nakayama I, et al. Aldosterone inhibits nitric oxide synthesis in rat vascular smooth muscle cells induced by interleukin-1 beta. Eur J Pharmacol 1995:290:69-73.

34. Sun $\mathbf{Y}$, Zhang J, Lu L, et al. Aldosterone-induced inflammation in the rat heart : role of oxidative stress. Am J Pathol 2002;161:1773-81.

35. Blacher J, Amah G, Girerd X, et al. Association between increased plasma levels of aldosterone and decreased systemic arterial compliance in subjects with essential hypertension. Am J Hypertens 1997;10(12 Pt 1):1326-34.

36. Robert V, Van Thiem N, Cheav SL, et al. Increased cardiac types I and III collagen mRNAs in aldosterone-salt hypertension. Hypertension 1994;24:30-6. 